University of Nebraska - Lincoln

DigitalCommons@University of Nebraska - Lincoln

2006

\title{
Magnetism of Fe clusters and islands on Pt surfaces
}

D. Repetto

MPI für Festkörperforschung, Stuttgart, Germany

Jan Honolka

Max-Planck-Institut für Festkörperforschung, honolka@fzu.cz

S. Rusponi

Institut de Physique des Nanostructures, Lausanne, Switzerland

H. Brune

Institut de Physique des Nanostructures, Lausanne, Switzerland

Axel Enders

University of Nebraska-Lincoln, a.enders@me.com

See next page for additional authors

Follow this and additional works at: https://digitalcommons.unl.edu/physicsenders

Part of the Physics Commons

Repetto, D.; Honolka, Jan; Rusponi, S.; Brune, H.; Enders, Axel; and Kern, Klaus, "Magnetism of Fe clusters and islands on Pt surfaces" (2006). Axel Enders Publications. 24.

https://digitalcommons.unl.edu/physicsenders/24

This Article is brought to you for free and open access by the Research Papers in Physics and Astronomy at DigitalCommons@University of Nebraska - Lincoln. It has been accepted for inclusion in Axel Enders Publications by an authorized administrator of DigitalCommons@University of Nebraska - Lincoln. 


\section{Authors}

D. Repetto, Jan Honolka, S. Rusponi, H. Brune, Axel Enders, and Klaus Kern 


\title{
Magnetism of Fe clusters and islands on Pt surfaces
}

\author{
D. Repetto, ${ }^{1}$ J. Honolka, ${ }^{1}$ S. Rusponi,${ }^{2}$ H. Brune, ${ }^{2}$ A. Enders, ${ }^{1}$ and K. Kern ${ }^{1}$
}

1. MPI für Festkörperforschung, Heisenbergstr. 1, 70569 Stuttgart, Germany

2. Institut de Physique des Nanostructures, EPFL, 1015 Lausanne, Switzerland

Corresponding author - A. Enders

\begin{abstract}
Clusters and islands of Fe atoms have been prepared by noble gas buffer layer assisted growth as well as by standard molecular beam epitaxy on Pt substrates. Xe buffer layers have been utilized to promote the formation of compact, relaxed Fe clusters with narrow size distribution. Without the Xe buffer, strained Fe islands with a characteristic misfit dislocation network are formed. Magnetization loops obtained by magneto-optical Kerr effect measurements reveal that inplane easy magnetization axis is only found for the relaxed clusters, pointing out the important role of epitaxial lattice deformations for the magnetic anisotropy.
\end{abstract}

\section{Introduction}

The study of small clusters has shown that fundamental properties, such as magnetic moments and anisotropy, electronic structure or chemical reactivity, are different from bulk behavior and dependent on the cluster size [1, 2]. While in free clusters such phenomena are commonly ascribed to the modified atomic coordination, clusters in contact with surfaces are also affected by the underlying substrate $[3,4]$. Thus, the deposition of clusters offers the opportunity to exert influence on the cluster properties, for instance, by exploiting electronic interactions or strain effects.

Several approaches have been developed to achieve controlled deposition of size-selected nanoclusters onto a substrate. On one hand, clusters can be formed in the gas phase and deposited on the substrate by soft-landing on a noble gas buffer layer [5]. On the other hand, compact clusters can be fabricated by molecular beam epitaxy (MBE) directly on the substrate if the surface has been pre-covered with a noble gas buffer layer to enhance the adlayer mobility $[6,7]$. But also without such a buffer layer the heteroeptitaxial growth of some materials results in the formation of separated islands, offering a third method to fabricate supported nanoclusters [9].

A challenging aim is to explore specifics in the cluster properties resulting from the preparation method. The scope of this work is to analyze and compare the structural and magnetic properties of Fe clusters on Pt prepared by noble gas as- sisted growth on one hand, and by heteroepitaxy on the other hand. It will be shown that the structure and the magnetic anisotropy of the clusters is strongly influenced by strain effects imposed by the lattice mismatch between cluster material and support.

\section{Sample preparation}

The experiments were performed in an ultrahigh vacuum (UHV) chamber system described in detail elsewhere [10]. Modifications were made on the sample holder to extend the accessible temperature range of the sample down to a base temperature of $35 \mathrm{~K}$. Flat $\mathrm{Pt}(111)$ and stepped $\mathrm{Pt}(997)$ single crystals were used as substrates for the growth of $\mathrm{Fe}$ structures. The substrates were prepared by cycles of $\mathrm{Ar}^{+}$ion sputtering and annealing to $870 \mathrm{~K}$. The preparation was finishedwhen sharp basic $(\mathrm{Pt}(111))$ and superstructure $(\mathrm{Pt}(997))$ diffraction spots were observed over the entire surface area by low energy electron diffraction (LEED). The substrate cleanliness was checked byAuger electron spectroscopy (AES). Fe films were deposited by using an electron beam evaporator. The deposition rate was calibrated by a quartz microbalance prior to each deposition.

Clusters of Fe of a few nanometer in diameter were fabricated on $\mathrm{Pt}(997)$ by noble gas assisted self assembly, as described in [6-8]. The atomic substrate steps of the Pt(997) surface separating terraces of (111) orientation are not expected to influence the cluster formation at low preparation temperatures. Films of two atomic layers (ML) and 4ML Fe have been deposited by MBE onto the substrate which was pre-covered by a Xenon layer at 35K.Already at this low temperature, the mobility of the metal atoms on Xe is sufficiently high to form small clusters [7]. Warming up the substrate to $90 \mathrm{~K}$ causes evaporation of the Xe layer. The clusters coalesce during the Xe sublimation and thus grow in size, until making contact with the surface. The final cluster size and the size distribution depends mainly on the initial thickness of the Xe layer and, to some extent, also on the Fe coverage.

The thickness of the Xe buffer layer is controlled by adjusting the Xe partial pressure and the exposure time. Assuming a sticking coefficient of 1 at $35 \mathrm{~K}$ a gas flow of 5.5 Langmuir (1 
$\mathrm{L}=10^{-6}$ Torr $\left.\times 1 \mathrm{~s}\right)$ leads to the formation of $1 \mathrm{ML}$ Xe $(1 \mathrm{ML}=$ $10^{15}$ atoms $/ \mathrm{cm}^{2}$ ).

The advantages of this preparation methods are that (i) clusters of almost any material can be prepared without the limitations usually associated with epitaxy, such as wetting phenomena, surface mobility, and other perturbations by the substrate, and (ii) the cluster fabrication does not require experimental equipment beyond the standard tools commonly used for MBE growth.

Auger electron spectroscopy yields integral information about the result of each individual preparation step. The low energy Auger lines of $\mathrm{Pt}(64 \mathrm{eV})$ and $\mathrm{Fe}(47 \mathrm{eV})$ are most sensitive to morphological differences of nanostructures. A characteristic AES spectra of the clean Pt substrate is shown in Figure 1a. After depositing $100 \mathrm{~L} \approx 18 \mathrm{ML}$ of $\mathrm{Xe}$ at $35 \mathrm{~K}$ substrate temperature only the MNN Auger line of $\mathrm{Xe}$ at $544 \mathrm{eV}$ is visible (Figure 1b). The Xe layer is desorbed by gradually warming up the sample to $100 \mathrm{~K}$. The Xe partial pressure in the UHV chamber reaches its maximum at $78 \mathrm{~K}$ substrate temperature. During the Xe desorption the Fe becomes very mobile and coalescence of clusters occurs.

Without the Xe buffer layer the Fe is found to grow in the Volmer-Weber growth mode at substrate temperatures of $T_{\mathrm{S}}$ $=300 \mathrm{~K}$ and below [11]. Thus, islands of Fe are obtained with a size depending on the nominal Fe thickness. The Auger spectra in Figures 1 c\&d are observed on Fe clusters formed by 2ML $\mathrm{Fe} / 100 \mathrm{~L} \mathrm{Xe/Pt} \mathrm{(c),} \mathrm{and} \mathrm{on} \mathrm{epitaxial} \mathrm{2ML} \mathrm{Fe/Pt} \mathrm{(d),} \mathrm{respectively.} \mathrm{It}$ is clearly seen that (i) the intensity of the Pt peak at $64 \mathrm{eV}$, as well as the MNN lines at $168 \mathrm{eV}$ and $237 \mathrm{eV}$ are larger in (c), and (ii) the intensity ratio of the lines $\mathrm{Fe}_{47} \mathrm{eV} / \mathrm{Pt}_{64} \mathrm{eV}$ is larger for the Fe islands in (d). These findings are consistent with the picture that small Fe clusters covering only a fraction of the Pt surface are formed on the Xe layer, while extended Fe islands covering substantially larger areas of the Pt surface are formed without Xe.

\section{Results}

\subsection{Fe clusters}

Magnetization loops of the Fe structures have been recorded in-situ by magneto-optical Kerr effect measurements (MOKE) in polar and longitudinal geometry. The MOKE measurements were done in a temperature range between $40 \mathrm{~K}$ and $300 \mathrm{~K}$, after desorption of the Xe at 90-100 K. Longitudinal MOKE loops of clusters formed of 2 ML Fe and 4 ML Fe on $100 \mathrm{~L} \mathrm{Xe}$ are shown in Figures 2 a\&b. For clusters of $2 \mathrm{ML}$ Fe, $\mathrm{s}$-shaped magnetization loops with no remanence are found at $43 \mathrm{~K}$. The clusters are not magnetically saturated at the maximum available in-plane field of $70 \mathrm{mT}$. Increasing the amount of Fe significantly changes the shape of the loops.Aligning the magnetic moments of the clusters of $4 \mathrm{ML}$ Fe by a field within the surface plane results clearly in remanent magnetization (Figure 2b). For both samples no magnetic signal could be detected in polar geometry. Based on these observations a preferred magnetization axis along the substrate surface is concluded for Fe clusters on Pt substrates.

The magnetic anisotropy of the clusters is thus clearly different from the anisotropy of a $2 \mathrm{ML}$ Fe film grown at $40 \mathrm{~K}$ without the Xe buffer layer. At such low growth temperatures, the mobility of the Fe atoms is suppressed and a highly disordered and defect-rich adlayer is formed. Despite this significant surface roughness, clear perpendicular magnetization is found by polar MOKE, as shown in Figure 2c.

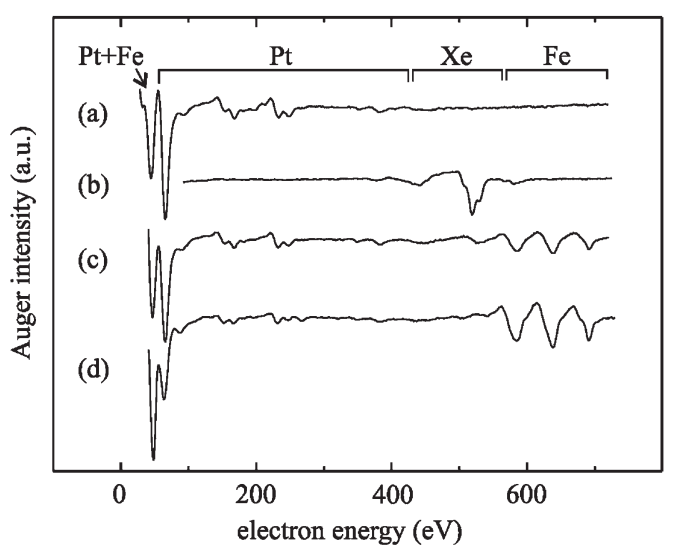

Figure 1. Auger spectra taken during different stages of the cluster preparation. (a) clean $\mathrm{Pt}$, (b) $\mathrm{Pt}+100 \mathrm{~L} \mathrm{Xe}$, (c) clusters of Fe after evaporation of $\mathrm{Xe}$, (d) epitaxial layer of $2 \mathrm{ML} \mathrm{Fe} / \mathrm{Pt}(111)$.

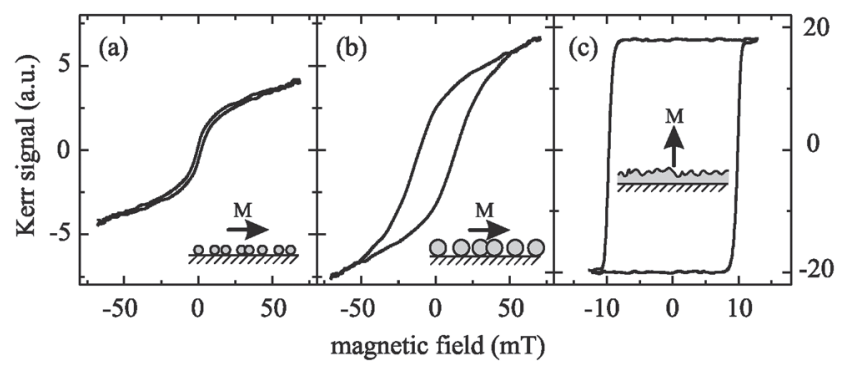

Figure 2. Magnetization loops observed at $\sim 40 \mathrm{~K}$ by (a) longitudinal MOKE on Fe clusters of $2 \mathrm{ML} \mathrm{Fe} / 100 \mathrm{~L} \mathrm{Xe/Pt}$, and (b) clusters of $4 \mathrm{ML} \mathrm{Fe} / 100 \mathrm{~L} \mathrm{Xe/Pt}$, (c) polar MOKE on $2 \mathrm{ML} F / \mathrm{Pt}$ grown at $41 \mathrm{~K}$.

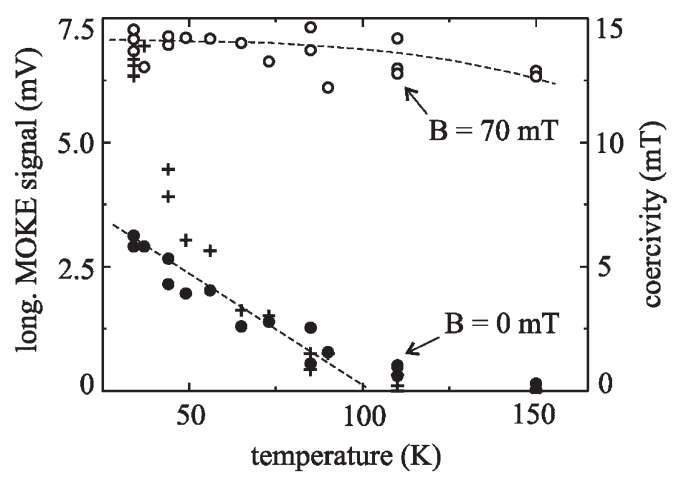

Figure 3. Temperature dependence of the magnetization of clusters of $4 \mathrm{ML}$ Fe measured in remanence $(\bullet)$ and at $70 \mathrm{mT}\left({ }^{\circ}\right)$, and the coercivity (+). The dashed lines are guides to the eye.

The investigation of the temperature dependence of the MOKE loops reveals further information about the magnetization of the cluster ensemble. The remanent MOKE signal $(\bullet)$ and the MOKE signal at $70 \mathrm{mT}\left({ }^{\circ}\right)$ is plotted for clusters of $4 \mathrm{ML} \mathrm{Fe}$ as a function of temperature in Figure 3. Additionally, also the temperature dependence of the coercive field is plotted (+). The data show that the remanent magnetization as well as the coercivity decrease with temperature and become zero at $T_{\mathrm{B}}=100 \mathrm{~K}$. In the same temperature range the MOKE signal at $H_{\text {max }}=70 \mathrm{mT}$ remains unchanged. 


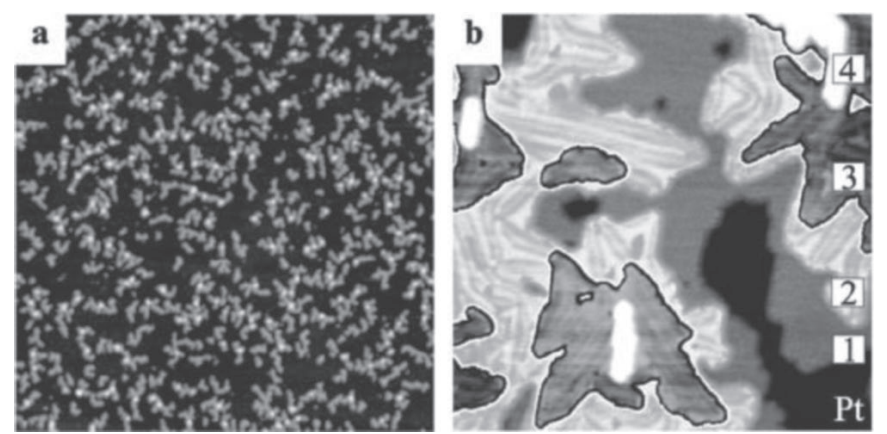

Figure 4. STM images of (a) $0.25 \mathrm{ML} \mathrm{Fe} / \mathrm{Pt}(111), T_{\text {growth }}=150 \mathrm{~K}$, image size $110 \times 110 \mathrm{~nm}^{2}$, and (b) $1.7 \mathrm{ML} \mathrm{Fe} / \mathrm{Pt}(111), T_{\text {growth }}=300 \mathrm{~K}$, image size $60 \times 60 \mathrm{~nm}^{2}$. The contrast in (b) has been adjusted to enhance the visibility of the misfit dislocations in the 2nd and 3rd Fe layer

The presented data are characteristic for superparamagnetic behavior of the deposited clusters. The observed temperature $T_{B}$ is thus interpreted as the blocking temperature of the clusters formed of $4 \mathrm{ML} \mathrm{Fe}$. A rough estimate of the mean cluster size can be made by

$$
V \approx 25 \times \frac{k T_{\mathrm{B}}}{E_{\mathrm{A}}}
$$

assuming bulk magnetic anisotropy, $E_{\mathrm{A}}=5.8 \times 10^{4} \mathrm{~J} / \mathrm{m}^{3}$ and inserting the experimentally determined blocking temperature and the Boltzmann factor, $k$. Assuming clusters of spherical shape and bulk lattice parameters, (1) gives a cluster diameter of $10.5 \mathrm{~nm}$, containing $5.1 \times 10^{4} \mathrm{Fe}$ atoms. In analogy, for clusters formed of $2 \mathrm{ML}$ Fe a blocking temperature of $T \mathrm{~B} \cong 50$ $\mathrm{K}$ is measured, giving a cluster diameter of $\approx 8.3 \mathrm{~nm}$, or $2.5 \times$ $10^{4} \mathrm{Fe}$ atoms per cluster. One can see that doubling the nominal Fe thickness roughly doubles the average number of $\mathrm{Fe}$ atoms per cluster.

\subsection{Epitaxial 3-D Fe islands}

While the Fe preparation on noble gas buffer layers supports the formation of clusters independent of the substrate properties, the growth of Fe on the $\mathrm{Pt}(111)$ without Xe is significantly influenced by the growth dynamics and the lattice misfit to the substrate. Fe is found to grow in the Volmer-Weber mode for substrate temperatures of $T_{\mathrm{S}}=300 \mathrm{~K}$ [11]. This means that already for sub-monolayer coverage several open layers grow simultaneously, giving rise to three-dimensional Fe islands. The epitaxy of Fe on Pt has been studied by scanning tunneling microscopy (STM). Figure 4a shows the initial growth of $\mathrm{Fe}$ on $\mathrm{Pt}(111)$ at $T_{\mathrm{S}}=150 \mathrm{~K}$. The nucleation of small islands of irregular shape is observed. Already at this low coverage, the onset of nucleation of the second layer on top of the islands of the first Fe layer is visible. The islands grow with increasing coverage laterally, but also in height by forming more and more open Fe layers. The Fe growth does not change qualitatively for substrate temperatures of $T_{S}=300 \mathrm{~K}$. An STM image characteristic for 1.7 ML Fe grown at $300 \mathrm{~K}$ is shown in Figure $4 \mathrm{~b}$. At this coverage, three-dimensional islands with ramified shape are found. The analysis of the STM images reveals that the first layer is not yet completed, but rather up to four layers are simultaneously opened and labeled in the figure. The islands of the second and third layer show character-

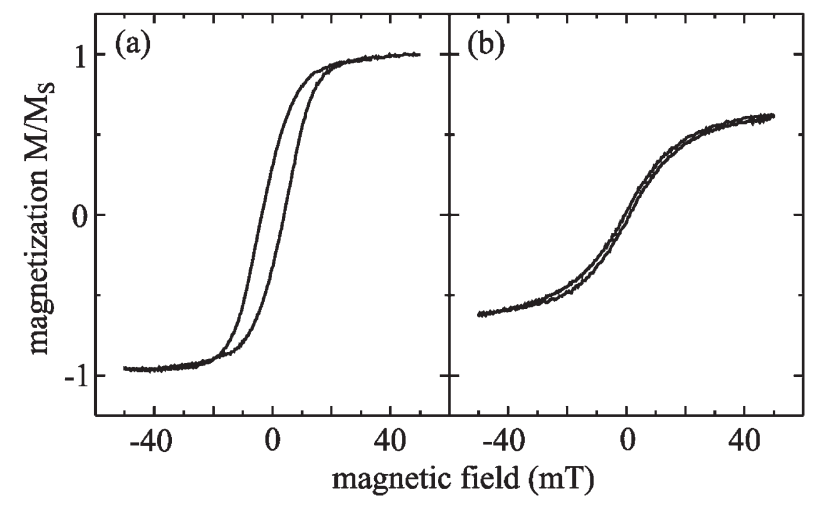

Figure 5. Polar MOKE magnetization loops of $2 \mathrm{ML} \mathrm{Fe} / \mathrm{Pt}(111)$, $T_{\text {growth }}=300 \mathrm{~K}$, measured at (a) $200 \mathrm{~K}$, and (b) $300 \mathrm{~K}$

istic corrugations. The visibility of the corrugations has been enhanced by expanding the gray scale of the image around the average gray value of these layers.

The corrugations are explained as the result of the relaxation of epitaxial strain. According to LEED investigation, the atoms of the first Fe layer occupy lattice sites provided by the $\mathrm{Pt}$, thus forming a pseudomorphic fcc(111) layer. Assuming a lattice constant of fcc Fe, $a_{\mathrm{fec}}^{\mathrm{Fe}}=3.59 \AA$ [12] and of Pt, $a_{\mathrm{Pt}}=3.92$ $\AA$, this implies a considerable epitaxial tensile strain of the first Fe layer of $9.2 \%$ ! The strain can significantly be lowered by inserting additional rows of Fe atoms in the second and third layer, giving rise to the observed surface corrugation. The strain relaxation provides the basis for a structural transition towards bcc at higher coverage [13].

The magnetism of the Fe islands has been investigated as a function of Fe thickness and temperature by MOKE. For a 2 ML Fe film on $\mathrm{Pt}(111)$ open magnetization loops are only found in polar MOKE geometry and for sample temperatures below $200 \mathrm{~K}$ (Figure 5a). Warming up the sample to $300 \mathrm{~K}$ results in s-shaped magnetization loops shown in Figure $5 \mathrm{~b}$. The MOKE analysis of various samples shows that (i) The easy magnetization axis of films thinner than $2 \mathrm{ML}$ is found to be perpendicular to the surface. (ii) The spin reorientation into the plane occurs gradually via a canted state in the thickness range of 2.2 $\pm 0.2 \mathrm{ML}$. (iii) Open polar magnetization loops are observed only if the substrate is cooled below $200 \mathrm{~K}$. At measurement temperatures of $300 \mathrm{~K}$ no remanent magnetization is observed along the surface normal [11].

\section{Discussion}

The results presented in the previous section show that Xe buffer layer assisted growth of Fe on Pt significantly influences the structural and magnetic properties of the Fe adlayer. The Xe layer promotes the formation of separated nanoclusters of Fe which are assumed to exhibit the unstrained bcc structure of bulk Fe prior to the contact with the Pt. The magnetization loops of the clusters show an easy axis within the substrate plane. The magnetic behavior is interpreted as superparamagnetism of a particle ensemble. On the other hand, the epitaxial growth of $\mathrm{Fe}$ on the $\mathrm{Pt}$ directly yields Fe islands of ramified shape and with visible lattice distortions due to the structural misfit to the substrate. The easy axis of the Fe islands is found to be perpendicular to the plane. 
The most striking difference between the Fe clusters and islands is, besides their specific morphologies, the lattice strain. Such epitaxial strain is known to contribute to the total magnetic energy via the magneto-elastic coupling constant, $B_{1}$. In order to apply such a phenomenological model commonly used for thin epitaxial films here, the balance between the magnetostatic energy,

$$
\Delta f_{\text {shape }}=1 / 2 \mu_{0} M_{\mathrm{S}}^{2}
$$

and the magneto-elastic energy,

$$
\Delta f_{\mathrm{ME}}=B_{1}\left(\varepsilon_{\perp}-\varepsilon_{||}\right) \text {. }
$$

has to be analyzed. Both expressions represent energy differences between out-of-plane and in-plane magnetization. Equation (3) gives negative values if perpendicular magnetization is preferred.

The magneto-elastic energy is calculated by using the value for $B_{1}$ found for strained Fe layers, $B^{\text {eff }}{ }_{1}=1 \times 10^{7} \mathrm{~J} / \mathrm{m}^{3}$ and assuming the in-plane strain of $\varepsilon_{\perp}=0.092$. The $B^{\text {eff }}{ }_{1}$ reflects the observation that epitaxial films show a strain dependent magneto-elastic coupling different from $B^{\text {bulk }}$. The value used here is an estimate based on experimentally determined $B_{1}^{\text {eff }}$ for epitaxial Fe films on W(100) and the calculated value using a strain model [14]. The calculation of the strain perpendicular to the surface, $\varepsilon_{\perp}$, requires a transformation of the strain tensor into film coordinates [15]. One obtains $\varepsilon_{\perp}=-0.184$. Using (3) a magneto-elastic energy of $-2.8 \times 10^{6} \mathrm{~J} / \mathrm{m}^{3}$ is calculated. This value provides an upper limit for the magneto-elastic energy, since the pseudomorphic strain of the first monolayer is assumed for the entire film. Yet, this simple model shows that the strain contribution alone is sufficient to defy the magnetostatic energy of $\Delta f_{\text {shape }}=1.9 \times 10^{6} \mathrm{~J} / \mathrm{m}^{3}$ !

The estimate predicts a perpendicular magnetization for fcc(111) Fe films due to strain and without consideration of surface anisotropy. Surface anisotropy contributions are usually significant and often dominate the magnetism of monolayer thin films. Surface and interface roughness are often found to influence the magnitude of the surface anisotropy [16]. Such contributions to the anisotropy arising from the film morphology can only be determined by a complex analysis for each individual system and are neglected in this estimate for simplicity.

Besides the dominating shape anisotropy for the relaxed clusters, also inter-particle interactions as well as particle-substrate interactions can be expected to be relevant for the observed in-plane magnetization. It is generally recognized that dipole-dipole interaction in cluster and nanodot assemblies can affect the magnetic behavior $[17,18]$. In addition, also indirect coupling between Fe nanodots through a $\mathrm{Cu}$ substrate promoting ferromagnetic in-plane ordering has been reported recently [19].
In summary, the magnetic properties of Fe clusters prepared by noble gas buffer layer assisted growth are compared to those observed on epitaxial, three-dimensional Fe islands. The clusters show superparamagnetic behavior and in-plane magnetic anisotropy. The easy magnetization axis of Fe islands perpendicular to the surface, on the other hand, can solely be explained by strain effects due to the lattice mismatch with the substrate. The advantage of the buffer layer assisted growth is hence that magnetic nanostructures can be prepared on substrate surfaces without the limitations and implications typically arising from the lattice mismatching in molecular beam epitaxy.

Acknowledgments - This project is supported by the Deutsche Forschungsgemeinschaft, SPP1153.

\section{References}

1. T. P. Martin, Phys. Rept. 273 (1996) 199

2. W. A. de Heer, Rev. Mod. Phys. 65, 611 (1993)

3. K.Wildberger, V.S. Stepanyuk, P. Lang, R. Zeller, P.H. Dederichs, Phys. Rev. Lett. 75, 509 (1995)

4. G. M. Pastor, J. Dorantes-Dávila, S. Pick, H. Dreyssé, Phys. Rev. Lett. 75, 326 (1995)

5. K. Bromann, C. Felix, H. Brune, W. Harbich, R. Monot, J. Buttet, K. Kern, Science 274, 956 (1996)

6. J. H. Weaver, G. D. Waddill, Science 251, 1444 (1991)

7. Ch. Haley, J. H. Weaver, Surf. Sci. 518, 243 (2002)

8. L. Huang, S. J. Chey, J. H. Weaver, Phys. Rev. Lett. 80, 4095 (1998)

9. O. Fruchart, M. Klaua, J. Barthel, J. Kirschner, Phys. Rev. Lett. 83, 2769 (1999)

10. D. Peterka, A. Enders, G. Haas, K. Kern, Rev. Sci. Instrum. 74, 2744 (2003)

11. D. Repetto, T. Y. Lee, J. Honolka, K. Kuhnke, A. Enders, K. Kern, S. Rusponi, H. Brune, submitted

12. F. Jona, P.M. Marcus, Crit. Rev. Surf. Chem. 4, 189 (1994)

13. H. Brune, K. Bromann, H. Röder, K. Kern, J. Jacobsen, P. Stoltze, J. Jacobsen, J. Norskow, Phys. Rev. B 52, R14 380 (1995)

14. A. Enders, D. Sander, K. Kirschner, J. Appl. Phys. 85, 5279 (1999)

15. D. Sander, Rep. Prog. Phys. 62, 809 (1999)

16. P. Bruno, J. Phys. F: Met. Phys. 18, 1291 (1988)

17. D. Kechrakos, K. N. Trohidou, Phys. Rev. B 58, 12169 (1998)

18. V. Novosad, K. Y. Guslienko, H. Shima, Y. Otani, S. G. Kim, K. Fukamichi, N. Kikuchi, O. Kitakami, Y. Shimada, Phys. Rev. B 65, 60402 (2002)

19. J. P. Pierce, M. A. Torija, Z. Gai, J. Shi, T. C. Schulthess, G. A. Farnan, J. F. Wendelken, E. W. Plummer, J. Shen, Phys. Rev. Lett. 92, 237201 (2004) 